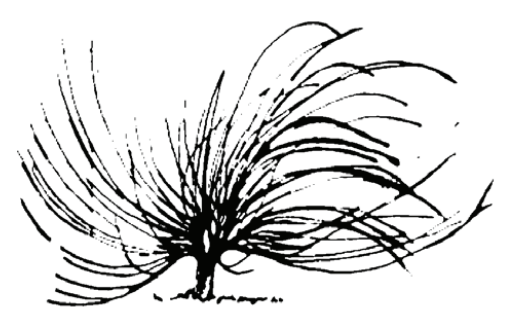

\title{
Aprender a Aprender: Implicaciones Psicopedagógicas del Uso del Conocimiento Estratégico en los Procesos de Aprendizaje
}

\author{
Giovanni Sánchez Chacón ${ }^{1}$ \\ Universidad Nacional \\ Heredia, Costa Rica \\ giovanni.sanchez.ch@gmail.com
}

\section{Resumen}

En el presente artículo, se exponen las bases conceptuales y metodológicas que proporcionan fundamentos para el análisis de las implicaciones psicopedagógicas del uso del conocimiento estratégico en los procesos de aprendizaje. Para estos efectos, se desarrollan conceptos claves en torno a este paradigma y se presentan algunas metodologías de trabajo orientadas a dicho fin.

Palabras clave: estrategias de aprendizaje, autonomía, autorregulación, conocimiento estratégico, metacognición, aprendizaje cooperativo

\section{(c) (1) (8) $\Theta$}

Recibido: 14 de julio de 2015-Aprobado: 4 de diciembre de 2015

1 Doctor en Psicología de la Educación, Cultura y Sistemas Semióticos. Universidad Autónoma de Barcelona, España. Posgrado en Psicología Evolutiva y de la Educación. Universidad Autónoma de Barcelona, España. Máster en Musicoterapia. Instituto Superior de Estudios Psicológicos, Barcelona, España. Máster en Psicopedagogía. Universidad Estatal a Distancia, UNED, Costa Rica. Licenciado en Ciencias de la Educación con énfasis en Didáctica de la Música. Universidad Nacional, Costa Rica. Bachiller en Música con énfasis en Educación Musical. Universidad Nacional, Costa Rica. 


\begin{abstract}
This article describes the conceptual and methodological framework that provides the basis for the analysis of psycho-pedagogical implications of strategic knowledge use in learning. For this purpose, key concepts are developed around this paradigm, and some working methods aimed at this purpose are presented.
\end{abstract}

Keywords: learning strategies, autonomy, self-regulation, strategic knowledge, metacognition, cooperative learning

\title{
Introducción
}

Aprender a aprender y aprender a pesar del sistema, representa en la actualidad, más que un reto, una necesidad para las nuevas generaciones de aprendices, tanto en contextos de educación formal como no formal. Esta situación se debe a que en ocasiones, ellos han sido educados a partir de metodologías de enseñanza que siguen priorizando el aprendizaje literal y mecánico de contenidos, en muchos casos aislados de sus contextos reales de aplicación.

Pensar en una educación que pretenda aportar algunas ideas al respecto, conlleva un fuerte compromiso con la reflexión en torno a algunos conceptos e ideas próximas a la psicología de la educación, y concretamente adheridos a una perspectiva de carácter sociocultural, la cual reconoce la influencia que ejerce la mediación social en los procesos de aprendizaje y en la conformación de los procesos psicológicos superiores implicados en la tarea de aprender a aprender.

Desde esta óptica, en el presente artículo, se exponen las bases conceptuales y metodológicas para el desarrollo del conocimiento estratégico, la autorregulación y la autonomía en el aprendizaje. En primer lugar, se presentan las bases teóricas necesarias que permitan comprender las implicaciones psicoeducativas de dichos conceptos. Posteriormente, se socializan algunas metodologías de trabajo, que de acuerdo con la investigación especializada, podrían promover progresivamente estas habilidades académicas, tanto en la educación formal como no formal y en el aprendizaje de cualquier disciplina. 


\section{Uso del conocimiento estratégico en los procesos de aprendizaje}

El uso del conocimiento estratégico, como paradigma aplicado a la educación, fue impulsado entre los años 80 y 90 por autores tales como Nisbet, Shucksmith, Pressley y Monereo. De acuerdo con (Nisbet y Shucksmith 1986), las estrategias de aprendizaje son concebidas como un conjunto de secuencias integradas de procedimientos planificados en función de un contexto de aprendizaje, con el propósito deliberado de facilitar la adquisición, almacenamiento y utilización de la información.

Desde esta perspectiva, las estrategias de aprendizaje, no podrían asumirse desde un marco de referencia meramente instrumental ni ser concebidas como técnicas de estudio, las cuales se planifican de manera intencional y deliberada, tras un análisis global del contexto, objetivos y condiciones concretas de aprendizaje. A partir de estos principios y siguiendo a (Pozo, Monereo y Castelló 2004), el desarrollo del conocimiento estratégico aplicado al aprendizaje, requeriría de un análisis profundo de las diversas variables inmersas en un contexto educativo particular y llevar al estudiante a plantearse cuándo, cómo, por qué y en qué circunstancias, el uso de unas determinadas estrategias de aprendizaje podrían tornarse más eficaces que otras. Tal y como se ha señalado, es imprescindible el desarrollo de una percepción sistémica, que permita la toma de conciencia por parte del aprendiz, respecto a la totalidad de demandas implicadas en sus aprendizajes particulares. Con lo anterior, sin duda el mismo accede de manera intencional a una comprensión de carácter cada vez más global, holística e integradora de todas las posibles variables que giran en torno a la consecución de un objetivo de aprendizaje y que permitan establecer las mejores rutas de acceso al conocimiento.

\section{Dimensiones del conocimiento estratégico}

Como hemos visto, el uso de una estrategia de aprendizaje requiere planificación de procedimientos conscientes y toma de control acerca de las principales demandas implicadas en el proceso. De acuerdo con (Pozo, Monereo y Castelló, 2004), la puesta en marcha de una estrategia conlleva que el aprendiz controle la planificación, supervisión y evaluación de un plan de acción. Siguiendo a los mismos autores, 
a continuación se exponen algunas de las dimensiones vinculadas al uso estratégico del conocimiento.

a. Las metas del aprendizaje: deben estar dirigidas a la comprensión de nuevos conceptos o a la reconstrucción de redes conceptuales. Pueden estar establecidas tanto por el docente como por el estudiante. Además, es importante considerar que dichas metas deben ser planteadas en el marco de una plena compatibilidad con los procedimientos estratégicos que permitan el alcance de ellas. .

b. El grado de control y regulación: se refiriere a la conciencia acerca de las demandas, que en cada etapa del proceso, comporta el objetivo de aprendizaje a alcanzar. Dentro de esta dimensión, es importante resaltar la importancia de la transferencia progresiva del control, del docente hacia el estudiante.

c. Nivel de incertidumbre de la tarea de aprendizaje: en este sentido, la incertidumbre debe entenderse como el grado de novedad que la tarea de aprendizaje conlleva, ya que por lo general, cuanto más inusual, novedosa y compleja sea, mayor abordaje estratégico requerirá.

d. La complejidad de la secuencia de acciones: en este caso, las secuencias de acciones representan los pasos implicados en la consecución de un objetivo de aprendizaje. El reto en esta dimensión es hacer un uso deliberado de estrategias concretas que aproximen a dicho objetivo, independientemente, de la complejidad que este conlleve.

Con lo anterior, podemos señalar que los procesos de toma de decisiones implicados en el desarrollo del conocimiento estratégico, requieren estrictamente, como se ha señalado, de una percepción global e integradora de todas las posibles variables implicadas en la consecución de unos determinados objetivos de aprendizaje. Lo anterior exige el planteamiento de principios metodológicos de corte psicopedagógico, encauzados al desarrollo de la autorregulación y la autonomía en el aprendizaje, que sin duda promoverán a todo nivel un uso progresivo y consciente del conocimiento estratégico ante unas demandas concretas de aprendizaje. 


\section{Autorregulación y autonomía en el aprendizaje}

Una de las principales tareas de la educación actual, consiste en formar aprendices autónomos capaces de aprender a lo largo de la vida y de transferir esos aprendizajes a las diversas situaciones que enfrentarán en los múltiples escenarios de su vida cotidiana. Más allá de una formación académica basada en contenidos inflexibles, es preciso, que las nuevas políticas educativas, estén articuladas a partir de principios epistemológicos, axiológicos y ontológicos que difundan un tipo de educación, cuyas bases consoliden una plataforma que permita al estudiante desarrollar una serie de competencias para seguir aprendiendo a lo largo de la vida. Por eso, se requiere que el aprendiz, además de los contenidos curriculares propios de su disciplina, desarrolle otro tipo de competencias con las que pueda gestionar con mayor precisión su propio proceso de aprender a aprender. En dicha gestión, están involucrados procesos de autorregulación, los cuales de acuerdo con (Manrique, 2004) representan la posibilidad del estudiante de aprender a aprender, la cual es producto del desarrollo de una mayor conciencia de sus procesos cognitivos y metacognitivos. Por su parte, (Monereo, 2001) señala que ella podría estar orientada a la capacidad de controlar, monitorear y dirigir los procesos mentales empleados en la consecución de diversos objetivos de aprendizaje.

De lo anterior, se deriva la importancia del desarrollo de otros procesos psicológicos primarios previos a la autorregulación, los cuales mediados socialmente, proporcionarían las bases para la concreción de comportamientos relativamente autónomos ante una tarea de aprendizaje. Dichos procesos podrían estar relacionados con el desarrollo de metacognición, definida como los procesos mentales dirigidos a la toma de conciencia sobre el propio pensamiento y sobre los recursos que empleamos para pensar y aprender. Desde esta perspectiva, las habilidades metacognitivas asumirían en un primer momento la toma de consciencia sobre dichos procesos y la autorregulación permitiría redirigir, encauzar y cambiar el curso de las decisiones que se emplean ante una tarea de aprendizaje.

Desde esta óptica, el desarrollo de habilidades metacognitivas y comportamientos autorregulados, proporciona al aprendíz las competencias de autonomía necesarias para encarar el proceso de aprendizaje de manera más consciente y deliberada. (Pozo, Monereo y Castelló 2004) 
se refieren a la autonomía en el aprendizaje como aquella facultad que le permite al estudiante tomar decisiones que le conduzcan a regular su propio aprendizaje en función a una determinada meta y a un contexto o condiciones específicas de aprendizaje. Por su parte, (Monereo 2001) define la autonomía en el aprendizaje no como independencia, sino como la facultad que posee el aprendiz de tomar las decisiones que le permitan regular el propio aprendizaje y así aproximarlo a una determinada meta. Para estos efectos, es imprescindible la toma de conciencia de las condiciones específicas que forman parte del contexto de aprendizaje. Desde esta misma óptica (Bornas, 1994), señala que un aprendiz autónomo es aquel cuyo sistema de autorregulación funciona de modo que le permite satisfacer exitosamente tanto las demandas internas como externas que le plantean unos determinados objetivos de aprendizaje.

Dicho lo anterior, se infiere que una educación que promueva la autorregulación y la autonomía en el aprendizaje, sugiere un posicionamiento didáctico alejado de percepciones reduccionistas. Por el contrario, estos principios psicoeducativos, están altamente comprometidos con el pensamiento crítico, estratégico y sistémico, que sugieren formas de enseñanza que permitan al aprendiz comprender, desde una perspectiva sistémica y a partir de una noción de ecología profunda (Naess, 1973) las demandas implicadas en un contexto de aprendizaje particular. El desarrollo de esta mirada global e integradora que los procesos de aprendizaje comportan es crucial en materia de desarrollo del conocimiento estratégico en un aprendiz, ya que a partir de estas nociones, se establecerían las condiciones para la toma de decisiones cada vez más ajustadas a la complejidad que un determinado contexto de aprendizaje plantea.

Por esta razón, resulta importante también servirse de una serie de principios metodológicos, que a través de pautas generales, establezcan posibles rutas que permitan llevar a la práctica dichos principios en contextos de enseñanza y aprendizaje diversos. A continuación, se comparten algunas ideas que la literatura especializada señala al respecto.

\section{Aspectos metodológicos de corte psicopedagógico}

Desde una perspectiva psicopedagógica, adherida a la tradición sociocultural del aprendizaje, (Monereo, 2001) señala una serie de principios y metodologías de trabajo, cuyo uso consciente y deliberado podría 
colaborar en el desarrollo progresivo de habilidades que potencien la autorregulación y la autonomía en los procesos de aprendizaje. Dichas pautas pretenden ser concebidas como un conjunto de orientaciones flexibles, dinámicas y contextuales, que puedan ser transferidas con facilidad a los procesos de enseñanza y aprendizaje de cualquier disciplina.

Desde este marco de referencia, se argumenta que todo proceso de aprendizaje, debe planificarse a nivel general tomando en consideración tres fases, dentro de las que se proponen orientaciones generales que permitan potenciar la autorregulación y la autonomía en el aprendizaje, al propiciar una transferencia progresiva del control del docente hacia el estudiante y comprometiendo gradualmente al último en cada una de las etapas del proceso, hasta llegar a alcanzar comportamientos autorregulados y autónomos. A continuación, la información presentada en la (figura 1), pretende aportar una mayor comprensión de dichos principios, posteriormente, con la intención de no caer en visiones de carácter instrumental, se presenta una reseña general de los métodos señalados por (Monereo, 2001), la cual fuera de todo tecnicismo, sugiere al lector pautas generales que le permitan hacer un uso flexible y creativo de estas ideas en función de los contextos de enseñanza y aprendizaje en que este participe.

\section{Figura 1: Fases para el desarrollo de la Autorregulación} y la Autonomía.
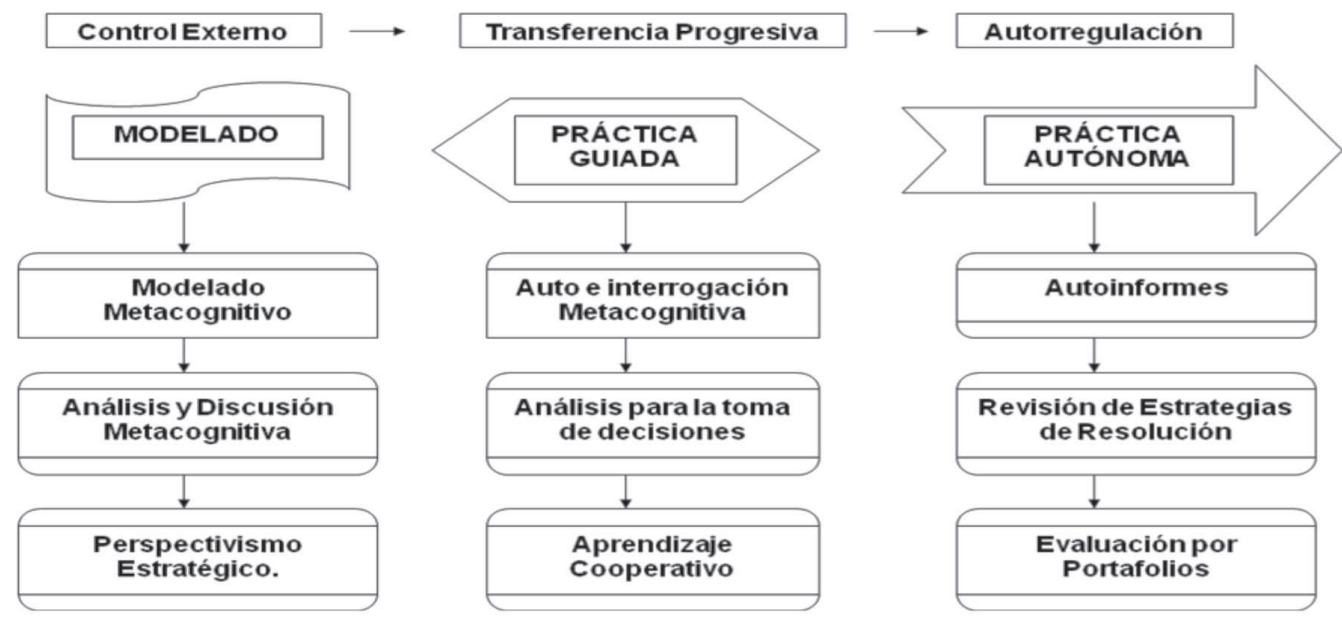

Fuente: (Monereo, 2001). 


\section{Fase I: Presentación de la estrategia (Modelado)}

En esta etapa, la función del docente es actuar como modelo, ejemplificando y haciendo demostraciones de carácter procedimental para apropiarse de contenidos de aprendizaje concretos. (Monereo, 2001) refiriéndose a esta fase, sugiere que ella aporta al estudiante una guía con posibles rutas de resolución ante una tarea compleja, concretamente, en momentos críticos del proceso. Con el propósito de lograr este objetivo, el mismo autor plantea algunas metodologías, a partir de las cuales podrían diseñar estrategias de enseñanza y aprendizaje orientadas al desarrollo de la autonomía y la autorregulación, las cuales se describen a continuación:

\section{a. Modelado Metacognitivo}

Dentro de esta metodología, se promueven el uso de estrategias de enseñanza en donde un experto actúa como modelo, explicando y justificando las estrategias que el mismo pone en marcha y lo que este piensa y hace en momentos críticos del proceso de resolución de una determinada tarea de aprendizaje.

\section{b. Análisis y Discusión Metacognitiva: (Autoevaluación Dirigida)}

Dentro del análisis y discusión metacognitiva, se suelen plantear estrategias de enseñanza que estimulen la autoevaluación de las acciones que un estudiante ha seguido en todas las etapas implicadas en la consecución de un objetivo de aprendizaje. Desde esta perspectiva, al finalizar una tarea de aprendizaje, se le plantea al estudiante una pauta que le permita examinar detenidamente lo que hicieron y pensaron antes, durante y después del proceso de resolución de un problema complejo. Posteriormente, se concede un espacio para que ellos reflexionen en torno a que modificarían del proceso seguido.

\section{c. Perspectivismo Estratégico}

Definida por (Monereo, 2001) como la habilidad de una persona para comprender cuál ha sido la estrategia empleada por un sujeto para resolver un determinado problema, en este caso un problema de 
aprendizaje. Dentro de estos métodos, se asumen estrategias en donde usualmente se solicita a un estudiante, observar cómo enfrenta un compañero la resolución de un problema complejo de aprendizaje. El primero observa a su compañero durante todo el proceso y al terminar la actividad, intenta socializar con este, cuáles han sido, desde su punto de vista, las estrategias empleadas en la resolución de la tarea o actividad en cuestión. Dentro de esta dinámica, el segundo estudiante, tendrá la oportunidad, en el marco de un diálogo constructivo, de confirmar o desmentir la apreciación de su compañero. Luego se intercambian los papeles siguiendo el mismo proceso. Por último, de manera conjunta ambos estudiantes proponen una serie de estrategias para aproximarse a dicho objetivo de aprendizaje desde una perspectiva más estratégica.

\section{Fase II: Práctica Guiada de la Estrategia}

En esta etapa, el docente intenta ceder progresivamente el control del proceso hacia los estudiantes, empleando estrategias que comprometan a ellos gradualmente con su propio proceso. Algunas metodologías que caracterizan esta fase y que han sido señaladas por la literatura especializada se señalan a continuación:

\section{a. Técnicas de Aprendizaje Cooperativo}

Dentro de esta fase, se promueve el uso de estrategias de enseñanza y aprendizaje vinculadas a la cooperación y el trabajo en equipo, como iniciativa que permite ir transfiriendo el control del proceso de aprendizaje, del docente hasta el estudiante. De acuerdo con (Durán y Vidal 2004), algunas de las metodologías sugeridas por la literatura proponen el uso del método Puzzle, la Tutoría entre Iguales, Grupo de Investigación, Enseñanza Recíproca, entre otros. A continuación se expone una breve reseña de cada una.

\section{- $\quad$ Método Puzzle (Rompecabezas)}

Para el desarrollo de este método, es necesario establecer dos tipos de agrupamientos: el equipo base (heterogéneo) y el de expertos (homogéneo). La dinámica de trabajo se desarrolla en los siguientes pasos: 
a. Se asigna de manera aleatoria al estudiantado en el grupo base, al cual se le encomienda la resolución de un problema, posteriormente se le asigna a cada participante un subtema clave para la resolución de dicha actividad, en el cual tendrán que convertirse en expertos.

b. Los estudiantes pasan del grupo base al grupo de expertos, en este todos los miembros tienen asignado el mismo tema en el que tendrán que especializarse.

c. Posteriormente, se regresa al grupo base, en donde los estudiantes deberán exponer la parte de los conocimientos que han adquirido en actividades resueltas en el grupo de expertos. Esta estructura permite que el aporte de cada miembro del equipo base se vuelva indispensable para la resolución de la actividad, la cual es evaluada mediante una calificación de equipo.

\section{- $\quad$ Tutoría entre Iguales}

De acuerdo con (Duran y Vidal, 2004), la tutoría entre iguales consiste en un método de aprendizaje cooperativo basado en la creación de parejas de alumnos con una relación asimétrica en cuanto a nivel de competencia y unos objetivos en común conocidos y compartidos por todos los miembros del equipo, los cuales se consiguen a través de un marco de relación exteriormente planificado. Dentro de las iniciativas de aprendizaje vinculadas a esta metodología, se benefician ambos estudiantes, inclusive el tutor, debido a la posibilidad que este último posee de aprender mientras enseña a su compañero (Topping, 1996). Desde esta perspectiva, se recomienda que los y las docentes establezcan un marco de interacción altamente estructurado, que a su vez, permita un progresivo control de este por parte de los alumnos, ajustando esa estructura interactiva propuesta, según las condiciones del contexto de aprendizaje.

Dentro de la tutoría entre iguales existen diversos formatos, los cuales pueden plantearse de diversas formas, ya sea en función de la edad y de la continuidad de los roles, como es el caso de la tutoría entre alumnos de distinta edad y de la misma edad. Dentro de este marco, también se pueden desarrollar formas de tutoría recíproca, en donde los $\mathrm{y}$ las estudiantes suelen alternar el rol de tutor y tutorado en cada sesión de trabajo. 


\section{- Investigación de Grupo}

Este método fue creado por (Sharan y Sharan, 1992). Al respecto, (Echeta y Martín 1990) establecen que este método podría ser uno de los más complejos y el que mejor responde a la filosofía de los grupos cooperativos. Lo anterior debido a que por su naturaleza podría aportar una mayor variedad de experiencias de aprendizaje que el resto de los métodos adscritos a este marco conceptual.

Dentro de las estrategias de enseñanza y aprendizaje vinculadas a este método, el grupo-clase trabaja como una comunidad de investigación en torno a un tema específico, el cual es dividido en distintos subtemas o líneas concretas de investigación, los cuales son asignados a diversos subgrupos. Cada subgrupo investigará a profundidad el subtema de investigación asignado y luego socializará sus resultados con el resto de la clase. Es importante destacar que todos estos trabajos han de estar acompañados de instrumentos de evaluación unidireccional docente y de coevaluación que permitan la toma de conciencia por parte de docente y estudiantes acerca del proceso seguido.

\section{- Enseñanza Recíproca}

Este método fue diseñado por (Palincsar y Brown 1984) para la resolución de actividades de comprensión lectora. Dentro de las estrategias de enseñanza y aprendizaje vinculadas a este, cada estudiante es responsable de la realización de una tarea distinta, relacionada con alguna operación cognitiva necesaria para la comprensión lectora. Dentro de esta dinámica, cada miembro del grupo realiza una función distinta, pero complementaria a la del resto de sus compañeros y en donde se recomiendan equipos de máximo cuatro estudiantes, en los que se distribuyen sus roles de la siguiente manera:

a. Un estudiante lee el texto.

b. Otro simultáneamente toma apuntes de las ideas principales.

c. El siguiente redacta preguntas o interrogantes interesantes que se desprenden del tema.

d. El último intentará anticipar las posibles temáticas que el autor tratará en las siguientes páginas o capítulos, así como el eventual desarrollo de los acontecimientos que en la lectura se narran. 
Concluida la dinámica y en función del rol cumplido por cada estudiante, se discute en torno a las ideas principales de la lectura y a las conclusiones obtenidas de ella. Esta dinámica podría realizarse por cada página o capítulo que los estudiantes lean, esto podría variar en función de las características generales de los textos asignados.

\section{b. Interrogación y Autointerrogación Metacognitiva}

Dentro de este método, se distinguen estrategias de enseñanza y aprendizaje orientadas al diseño de pautas con las principales demandas implicadas en la resolución de problemas de aprendizaje complejos. Dichas pautas orientan la reflexión sobre dicho problema, por ende, la toma de decisiones que permita la ejecución de procedimientos estratégicos durante el proceso de resolución. Dicho proceso puede ser elaborado también por el propio estudiante o por un compañero tutor.

\section{c. Análisis para la toma de decisiones}

Dentro de esta metodología, se planifican estrategias que implican un mayor nivel de elaboración que el anterior método, al extraerse de una temática compleja de aprendizaje, aquellos datos concretos que son relevantes para tomar decisiones y con los que eventualmente se podría resolver dicho problema. Este método requiere el seguimiento de las siguientes tres fases:

a. Identificación y destacado de los datos fundamentales.

b. Organización de los datos seleccionados en algún sistema de representación.

c. Deducción de algún principio o ley que establezca alguna relación causal entre los datos.

\section{Fase III. Práctica Autónoma de la Estrategia}

Esta fase se caracteriza por el fuerte compromiso de lograr que el estudiante desarrolle autonomía en su aprendizaje. En esta etapa, el mismo discente debe ser consciente de su propio proceso de aprendizaje y de las demandas a nivel de autorregulación que dicho proceso 
implica. Para el logro de estos fines, a manera de orientaciones generales, (Monereo, 2001) sugiere el uso de las siguientes metodologías:

\section{a. Autoinformes}

Los autoinformes suponen exposiciones ordenadas de manera oral o escrita, de la forma en que un estudiante percibe o ha percibido una tarea de aprendizaje y del conjunto de decisiones que se han tomado en su resolución. Estos se desarrollan en función de las demandas que impliquen la consecución de un objetivo de aprendizaje y pueden ser "prospectivos", - administrados previamente al análisis de la problemática profesional planteada - o retrospectivos, - administrados a posteriori -.

\section{b. Revisión de la Estrategia}

En este tipo de métodos, se desarrollan estrategias orientadas hacia la autoevaluación de la propia planificación del aprendizaje. Su implementación consta de los siguientes tres pasos:

a. Previo a la resolución de un problema, el estudiante planifica el tipo de estrategias que desde su punto de vista, le ayudará a resolver dicha problemática.

b. Valora la eficacia de dicha estrategia a partir de los resultados obtenidos.

c. A partir de dicha valoración, introduce los cambios pertinentes y aplica la estrategia de nuevo.

\section{c. Portafolios de Evidencias}

Dentro de este método, se apuesta por una mayor interiorización de los criterios de evaluación empleados por el docente. Durante el desarrollo de la asignatura, se construye paralelamente un portafolio que contengan trabajos, tareas, producciones y en general cualquier tipo de evidencias de aprendizaje generadas en el contexto de la asignatura. Este portafolio estará estructurado en carpetas y los productos mencionados serán clasificados en función de los objetivos académicos establecidos en el programa del curso o planificación desarrollada por el o la docente. 
Cabe rescatar que estos métodos de trabajo, pretenden ser asumidos como principios o pautas generales de trabajo, cuyo uso estratégico y de carácter situado, podría generar estrategias de enseñanza $\mathrm{y}$ aprendizaje que aporten nuevas vías en la tarea de optar por un tipo de mediación pedagógica que promueva la autorregulación, el uso del conocimiento estratégico, el desarrollo de la autonomía y la formación aprendices permanentes.

\section{Conclusiones}

Como hemos visto, en todos los objetivos de aprendizaje, sin importar la disciplina a la que se adscriben, están implícitos una serie de exigencias de carácter cognitivo, procedimental y condicional, que resulta importante visibilizar desde una perspectiva sistémica o global. Los principios acá compartidos están orientados al desarrollo del conocimiento estratégico y formas de autorregulación que permitan formar aprendices autónomos y permanentes capaces de aprender a lo largo de la vida.

Dentro de esta perspectiva, es importante destacar la urgencia de apostar por una enseñanza explicita de competencias que permitan al estudiante aprender a aprender. En este marco específico, más que iniciativas individuales de cada docente, es preciso el planteamiento de políticas educativas que incorporen paralelamente a la enseñanza de contenidos curriculares, la enseñanza explícita de procedimientos estratégicos que permitan al aprendiz aproximarse de manera más certera a las metas y objetivos de aprendizaje propuestos. Lo anterior podría visibilizarse inicialmente, apostando no solo al desarrollo del conocimiento conceptual, procedimental y actitudinal tradicionalmente conocidos, sino también hacer explícita la relevancia del conocimiento condicional o estratégico implicado en toda tarea de aprendizaje.

Lo anterior recalca el interés, también en materia de política educativa, de la enseñanza explicita de aspectos de carácter psicopedagógico que desarrollen en el estudiante destrezas vinculadas a la metacognición, y a la autorregulación del aprendizaje. Lo dicho sin duda influirá positivamente en la formación de aprendices autónomos y competentes en la tarea de aprender a aprender, independientemente de los retos de aprendizaje que ellos enfrenten, tanto en procesos de educación 
formal como no formal o en otros niveles, situaciones o trayectorias personales de aprendizaje que la vida misma les demande.

\section{Referencias bibliográficas}

Bornas, X. (1994). La autonomía personal en la infancia. Estrategias cognitivas y pautas para su desarrollo. España, Siglo XXI editores.

Duran, D.; Vidal, V. (2004). Tutoría entre iguales: De la teoría a la Práctica. Barcelona: Graó.

Echeita, G. y Martin, E. (1990). Interacción social y aprendizaje. En A. Marchesi, C.Coll y J. Palacios. (Eds.). Desarrollo psicológico y educación III. Necesidades educativas especiales y aprendizaje escolar. Madrid: Alianza Psicológica.

Manrique, L. (2004). El aprendizaje autónomo en la educación a distancia. Recuperado de http://www.ateneonline.net/datos/55_03 Manrique_Lileya.pdf

Monereo, C. (2001). La enseñanza estratégica: enseñar para la autonomía, En C. Monereo. (Coord), Ser estratégico y Autónomo Aprendiendo, 11-27. Barcelona: Graó.

Naess, A. (1973). The shallow and the deep, long range ecology movements. Recuperado de http://www.alamut.com/subj/ideologies/ pessimism/Naess_deepEcology.html

Nisbet, J. y Shucksmith, J. (1986). Learning strategies. Londres: Routledge and Kegan Paul [Trad. cast.: Estrategias de aprendizaje. Madrid: Santillana, 1987].

Palincsar, A. Y Brown, A. (1984). Reciprocal teaching of comprehension-fostering and metacognitive strategies, Cognition and instruction, 1: 117-175.

Pozo, I., Monereo, C. y Castelló, M. (2004). El uso estratégico del conocimiento, En C. Coll, J. Palacios, y Á. Marchesi. (Comps), Desarrollo Psicológico y Educación, II. Psicología de la Educación, 211-233. Madrid: Alianza.

Sharan, S. (ed.) (1992). Handbook of cooperative learning methods. London: Praeger.

Topping, K. (1996). Effective Peer Tutoring in Further and Higher Education, Birmingham: SEDA paper. 